\title{
Top users consume half of health budget
}

$\mathrm{T}$ reating the top 5\% of users of Ontario's health system 662248 patients in 2011 accounted for more than half the provincial health budget, say a team of Toronto-based researchers who are probing data on so-called high-cost users.

These patients tend to be older, white women, with little postsecondary education, low household income and a median of eight chronic diseases, said Sara Guilcher of the Health System Performance Research Network at the University of Toronto. More than half of these patients are on 10 or more drugs.

The information on high-cost users, some of it in preliminary form, was presented by Public Health Ontario and the Institute for Clinical and Evaluative Sciences (ICES) at the Canadian Association for Health Services and Policy Research (CAHSPR) annual conference in Toronto May 14.

Guilcher told attendees that the most commonly used hospital and community care services were for the treatment of acute unplanned medical events, planned surgery and iatrogenic causes triggered by previous health care treatment. The iatrogenic events are the most expensive to treat, with costs averaging just over \$36 000 per event and consuming \$2.7 billion or $23 \%$ of total treatment costs for the top $5 \%$ of health care consumers.

"We were shocked to find that the iatrogenic causes cost so much," Guilcher said, while noting that the findings are preliminary. "A 35\% reduction would generate a billion dollars in savings."

But defining what causes these events is challenging, said Walter Wodchis, an adjunct scientist at ICES who directed Guilcher's study. "I think the best way to describe it is to say that something new happened to the patients after they were admitted to acute care that affected their length of stay." This could include a new diagnosis or medical issue, adverse effects of treatment or a medical error.

The burden of disease borne by the highest-cost users and the financial costs of caring for them, is an increasingly urgent issue, noted Laura Rosella, a scientist with Public Health Ontario who worked with Wodchis and Guilcher to link data from three cycles of the

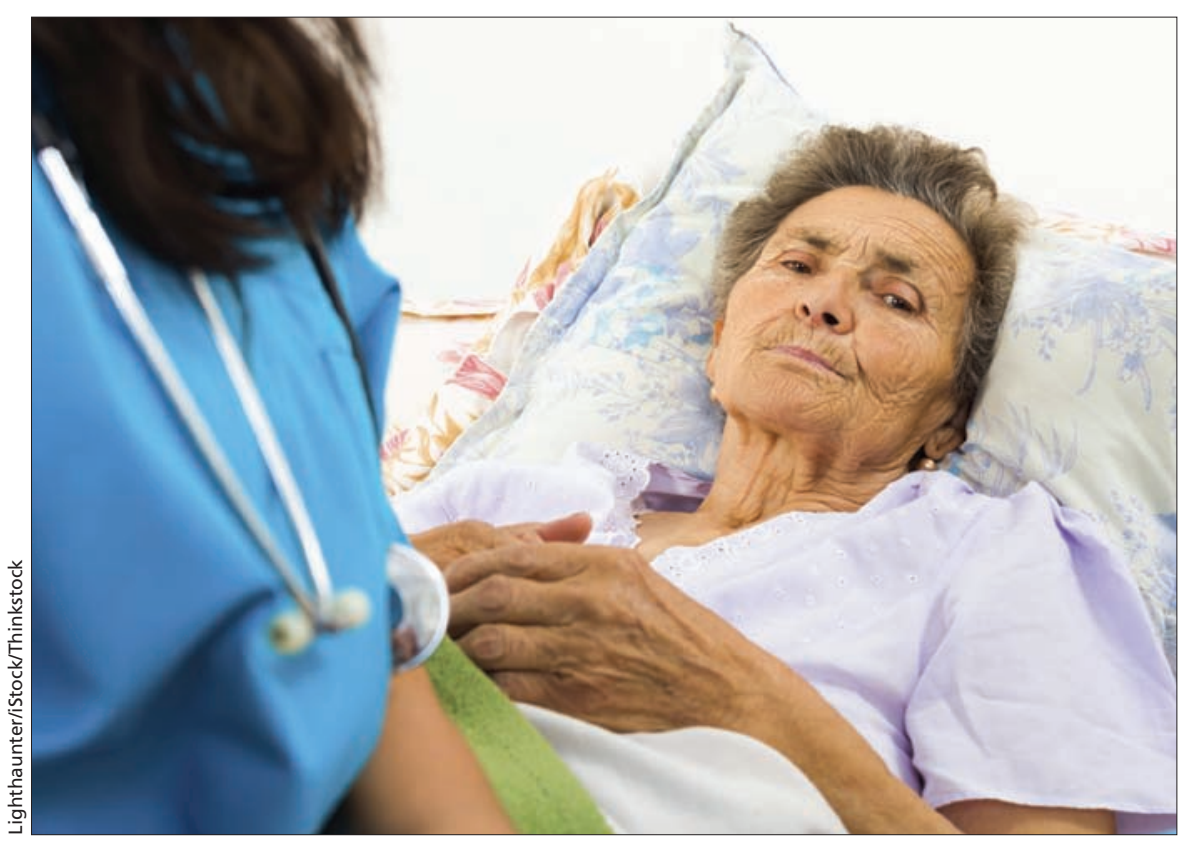

The top $5 \%$ of users of health care in Ontario tend to be older, white women with low income and comorbidities.

Canadian Community Health Survey for Ontario to medical claim data from the Ontario Health Insurance Plan to probe data on high-cost users. The data so far suggest this is a research area that offers insight into potential reforms to health care delivery, said Rosella. The aim is to "inform policies to improve population health and achieve sustainability of health services."

The top $1 \%$ of health care users, some 132200 people in Ontario, consume 25\% of the province's \$46-billion health budget, or an average of $\$ 53000$ per patient, Rosella noted. Hospital services expenditures account for $54 \%$ of this. Nearly a quarter of high-cost users are over 80 years old, $20 \%$ are obese and $32 \%$ are from low-income households. Obesity and a history of heavy smoking are associated with a $70 \%$ increased risk for becoming a high-cost user of health care. Lack of access to a family physician also greatly elevates the risk, Rosella noted.

Another study found that the top 1\% of high-cost users tend not to own their homes and to live in high unemployment areas, said Tiffany Fitzpatrick, an epidemiologist with Public Health Ontario. Her study concluded that food insecurity was very strongly associated with highcost health care use. Over a third of these patients come from low-income households, and about 56\% come from below middle-income households. "The odds of belonging to a low-income, compared to high-income, household were nearly seven times greater" in high-cost users, Fitzpatrick noted.

In another unpublished study of children who are persistently high-resource users, Dr. Astrid Guttmann, a senior scientist at ICES working with Wodchis, found that 28642 children were in the top $1 \%$ of pediatric health-resource users in 2007, with a median cost of \$22 945 . Among these children, 38\% had neurological impairment, and home care use was the largest contributor to cost.

Intriguingly, says Rosella, high-cost users often enter into intensive care use abruptly, rather than gradually. Citing data suggesting that $45 \%$ of the top $1 \%$ of health care users were never previously in the top 5\% of users, Rosella explained that very few patients remain high-cost users for more than five years.

"The transitions do not always occur gradually," Rosella noted, before explaining that death is by far the most common outcome. - Paul Christopher Webster, Toronto, Ont.

CMAJ 2014. DOI:10.1503/cmaj.109-4809 